\title{
Molecular cloning and sequencing analysis of the interferon receptor (IFNAR-1) from Columba livia
}

\author{
CHAO LI, WEI SHAN CHANG
}

Shandong Agriculture University, Shandong, PR China

\begin{abstract}
Objective: Partial sequence cloning of interferon receptor (IFNAR-1) of Columba livia.

Material and methods: In order to obtain a certain length $(630 \mathrm{bp})$ of gene, a pair of primers was designed according to the conserved nucleotide sequence of Gallus (EU477527.1) and Taeniopygia guttata (XM_002189232.1) IFNAR-1 gene fragment that was published by GenBank. Special primers were designed by the Race method to amplify the 3'terminal cDNA.

Results: The Columba livia IFNAR-1 displayed $88.5 \%, 80.5 \%$ and $73.8 \%$ nucleotide identity to Falco peregrinus, Gallus and Taeniopygia guttata, respectively. Phylogenetic analysis of the IFNARI gene showed that the relationship of Columba livia, Falco peregrinus and chicken had high homology.

Conclusions: We successfully obtained a Columba livia IFNAR-1 gene partial sequence. Analysis of the genetic tree showed that the relationship of Columba livia and Falco peregrinus IFNAR-1 had high homology. This result can be used as reference for further research and practical application.
\end{abstract}

Key words: Columba livia, IFNAR-1, gene cloning, gene sequence analysis.

(Centr Eur J Immunol 2014; 39 (2): 159-164)

\section{Introduction}

Interferon was identified by Isaacs and Lindenmann during their seminal studies on virus interference 50 years ago. They reported that influenza virus-infected chick cells produced a secreted factor that transferred a virus-resistant state to previously uninfected cells. Importantly, the resistance was displayed against both the homologous inducing virus and heterologous viruses. The factor was designated interferon (IFN) because of its ability to interfere with virus growth [1]. Since then IFN has been implicated in a wide variety of biological functions, including anti-proliferative effects, induction of human leukocyte antigen (HLA), increase in natural killer (NK) activity and others [2, 3].

Interferon proteins are historically characterised as type I $(\alpha, \beta)$ or viral IFNs and type II $(\gamma)$ or immune IFN [4]. Type I IFN induces antiviral, anti-proliferation, and immunomodulatory effects through interaction with specific receptor complexes on the surface of target cells, which consists of two chains: The high-affinity ligand-subunit (IFN- $\alpha, \beta$ receptor $\alpha$-chain, IFNAR-1) and the unknown function subunit (IFN- $\alpha, \beta$ receptor $\beta$-chain, IFNAR-2) [5, 6]. Similarly to other IFN receptors, IFNAR-1 is a single transmembrane protein of the class II cytokine receptor family (CRF2), defined by structural similarities in the extracellular domain. Interferon receptor-mediated signalling leads to the activation of latent cytoplasmic factors, the signal transducers and activators of transcription (STAT) family of proteins, which are activated by a process that involves members of the Janus tyrosine kinase (JAK) family [7-9].

The objective of this study was to clone a sequence of IFANR- 1 gene and provide a basic understanding of the gene mutations between Columba livia and animals closely related to it through phylogenetic analysis. Physiological and biochemical property studies of the obtained IFANR-1 gene and protein were conducted to provide valuable reference for further research on the gene.

\section{Material and methods}

\section{Sample collection and tissue preparation}

Spleen samples were collected from Columba livia. The Columba livia was brought as a live, mature Columba livia from a common farm and kept under inspection for 5 days to be sure that it was free from any clinical infection, then the sample was harvested and stored at $-20^{\circ} \mathrm{C}$. 
Table 1. Conserved sequence amplification PCR and 3'RACE products using forward and reverse primer sequences

\begin{tabular}{lc}
\hline Primer name & Primer sequences \\
\hline A & 5'-GCTCAGATTGGTCCCCCTGA-3' \\
\cline { 2 - 2 } & 5'-CAGGAGGGCCAATTTCATTTGTT-3' \\
\hline 3'RACE 1 & 5'-GCACCAGACACTACCTATT-3' \\
\hline 3'RACE 2 & 5'-CTAGCGCTCCTGACTCAGCGGTTTTTTTTTTTTTTTTTT-3' \\
\cline { 2 - 2 } & 5'-ACTCCACAGAGTGGCTCAGTGT-3' \\
\hline
\end{tabular}

\section{Total RNA isolation extraction and synthesis of cDNA}

Total RNA samples were extracted from spleens using Trizol (TransGen), and the cDNA was obtained using the PrimScript RT reagent Kit (TaKaRa) according to the manufacture's protocol.

\section{RT-PCR, 3'RACE and sequencing}

A pair of homologous primers (Table 1A) was designed with DNASTAR 5.0 software in the conserved region of Gallus (EU477527.1) and Taeniopygia guttata (XM_002189232.1). All primers used in this study are

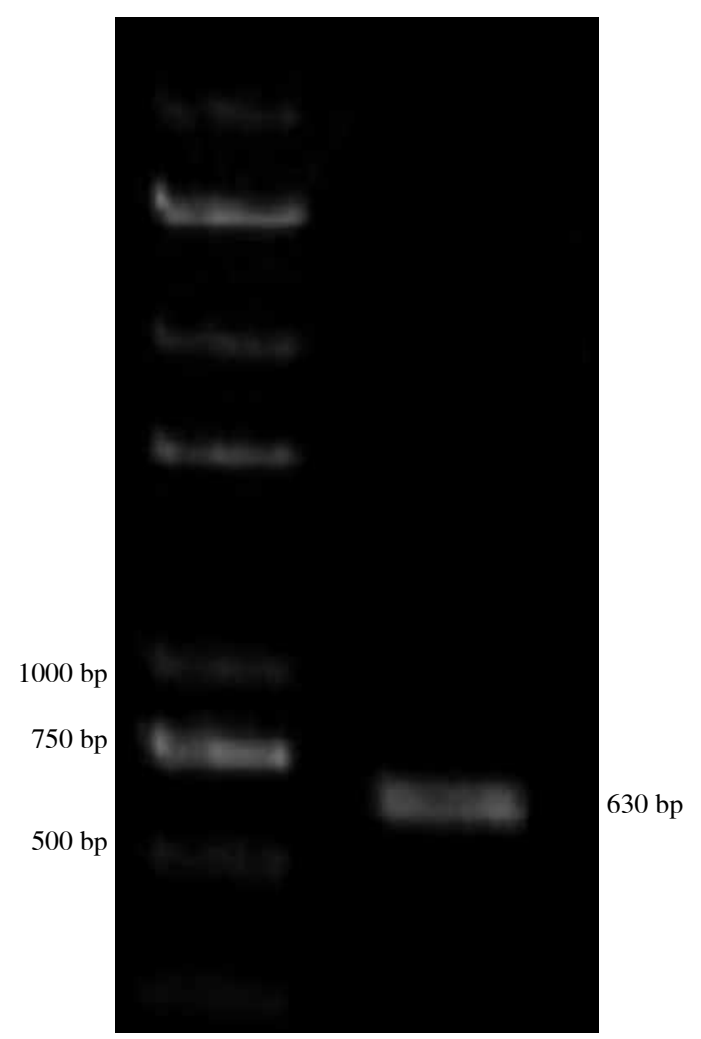

Fig. 1. RT-PCR amplification result of the conserved sequence listed in Table 1. With the primers, a cDNA fragment was amplified by RT-PCR using the first strand cDNAs as templates. The PCR was performed at $94^{\circ} \mathrm{C}$ for 5 minutes, followed by 30 cycles at $94^{\circ} \mathrm{C}$ for 45 seconds, $58^{\circ} \mathrm{C}$ for 40 seconds and $72^{\circ} \mathrm{C}$ for 1 minute, with a final extension at $72^{\circ} \mathrm{C}$ for 10 minutes, and then stopped at $4^{\circ} \mathrm{C}$. Polymerase chain reaction products were detected by electrophoresing $5 \mu \mathrm{l}$ aliquots through $1 \%$ agarose in $1 \times \mathrm{TAE}$ and purified by Agarose Gel DNA Extraction Kit (Shanghai Sangon Biotech Co., Ltd.). The products were cloned by into pEASY-T1 vector (TransGen) and sent to Shanghai Sangon Biotech Co., Ltd. for sequencing. Two pairs of specific primers (Table 1: 3'RACE 1 and 2) were designed

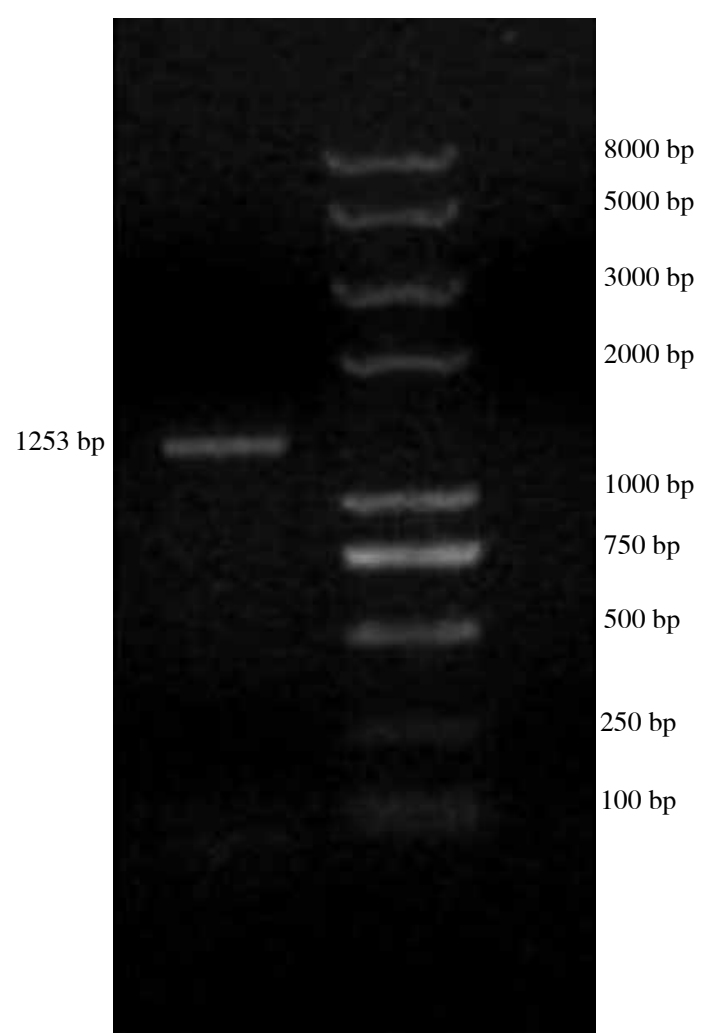

Fig. 2. Results of 3'RACE amplification 
according to the obtained sequences. In the 3'RACE and the second pair for the second round PCR products were analysed by electrophoresis in $1 \%$ agarose then purified and cloned into pEASY-T1 vector, detected and sequenced as mentioned above.

\section{IFNAR1 nucleotide sequence homology comparison and phylogenetic analysis}

Sequencing results of Columba livia IFNAR-1 and the nucleotide sequence of Gallus (EU477527.1), Falco peregrinus (XM_005234387.1), Melopsittacus undulatus (XM_005151790.1), Anas platyrhynchos (XM_005013847.1), Ficedula albicollis (XM_005037095.1), Taeniopygia guttata (XM_002189232.1), Papio Anubis (NM_001168783.1), Macaca mulatta (NM_001266513.2), Homosapiens (XM_005260964.1), Geospiza fortis (XM_005427794.1) that registered in GenBank were subtyped and put up for homologous analysis.

\section{IFNAR1 amino acid analysis}

Phylogenetic analysis was made using DNASTAR software. Sequence analysis of the predicted Columba livia IFNAR-1 protein translated from the nucleotide sequence of the Columba livia IFNAR1 fragment was performed using NCBI and ExPaSy software. Signal peptides were predicted using the Signal P 3.0 server. The domain structure of the Caprine protein was analysed on the SMART server. TMHMM Server v2.0 was used to predict transmembrane regions of IFNAR-1 protein.

\section{Results}

\section{Molecular cloning and analysis of Columba livia IFNAR-1}

A cDNA fragment of Columba livia IFNAR-1 of 630 bp (Fig. 1) was obtained from single RT-PCR that was

\begin{tabular}{c|c|c|c|c|c|c|c|c|c|c|c|c|}
\hline & $\mathbf{1}$ & $\mathbf{2}$ & $\mathbf{3}$ & $\mathbf{4}$ & $\mathbf{5}$ & $\mathbf{6}$ & $\mathbf{7}$ & $\mathbf{8}$ & $\mathbf{9}$ & $\mathbf{1 0}$ & $\mathbf{1 1}$ & \\
\hline $\mathbf{1}$ & & 88.5 & 73.8 & 80.5 & 74.0 & 55.8 & 55.9 & 86.0 & 56.2 & 73.8 & 80.3 & $\mathbf{1}$ \\
\hline $\mathbf{2}$ & 12.6 & & 72.1 & 79.9 & 71.5 & 56.4 & 55.9 & 86.3 & 57.1 & 70.3 & 81.6 & $\mathbf{2}$ \\
\hline $\mathbf{3}$ & 32.8 & 35.5 & & 68.3 & 89.9 & 46.3 & 46.2 & 71.3 & 49.3 & 88.2 & 66.7 & $\mathbf{3}$ \\
\hline $\mathbf{4}$ & 22.8 & 23.7 & 42.0 & & 68.6 & 54.5 & 54.3 & 77.9 & 55.5 & 67.2 & 79.2 & $\mathbf{4}$ \\
\hline $\mathbf{5}$ & 32.3 & 36.6 & 10.9 & 41.6 & & 48.9 & 49.1 & 71.5 & 49.4 & 90.5 & 69.6 & $\mathbf{5}$ \\
\hline $\mathbf{6}$ & 68.3 & 66.9 & 99.6 & 71.9 & 90.2 & & 90.6 & 55.4 & 95.4 & 46.6 & 51.4 & $\mathbf{6}$ \\
\hline $\mathbf{7}$ & 67.9 & 68.4 & 100.0 & 72.4 & 89.6 & 10.1 & & 54.7 & 98.9 & 47.9 & 52.2 & $\mathbf{7}$ \\
\hline $\mathbf{8}$ & 15.8 & 15.3 & 36.9 & 26.5 & 36.5 & 69.5 & 71.6 & & 57.1 & 68.7 & 81.3 & $\mathbf{8}$ \\
\hline $\mathbf{9}$ & 67.2 & 65.4 & 88.5 & 69.0 & 88.2 & 4.7 & 1.1 & 65.0 & & 49.5 & 57.0 & $\mathbf{9}$ \\
\hline $\mathbf{1 0}$ & 32.7 & 38.4 & 12.8 & 43.7 & 10.2 & 98.1 & 92.5 & 41.2 & 87.4 & & 68.4 & $\mathbf{1 0}$ \\
\hline $\mathbf{1 1}$ & 23.1 & 21.3 & $\mathbf{4 4 . 8}$ & 24.7 & 39.6 & 79.9 & 77.6 & 21.7 & 65.4 & $\mathbf{4 1 . 6}$ & & $\mathbf{1 1}$ \\
\hline & $\mathbf{1}$ & $\mathbf{2}$ & $\mathbf{3}$ & $\mathbf{4}$ & $\mathbf{5}$ & $\mathbf{6}$ & $\mathbf{7}$ & $\mathbf{8}$ & $\mathbf{9}$ & $\mathbf{1 0}$ & $\mathbf{1 1}$ & \\
\hline
\end{tabular}

\section{Columba livia.seq \\ Falco peregrinus.seq \\ Ficedula albicollis.seq \\ gallus.seq \\ Geospiza fortis.seq \\ Homo sapiens.seq \\ Macaca mulatta.seq \\ Melopsittacus undulatus.seq \\ Papio anubis.seq \\ Taeniopygia guttata.seq \\ Anas platyrhynchos.seq}

Fig. 3. Homology comparisons of nucleotide sequences among Gallus, Columba livia, Falco peregrinus and other animals IFNAR-1 from GenBank

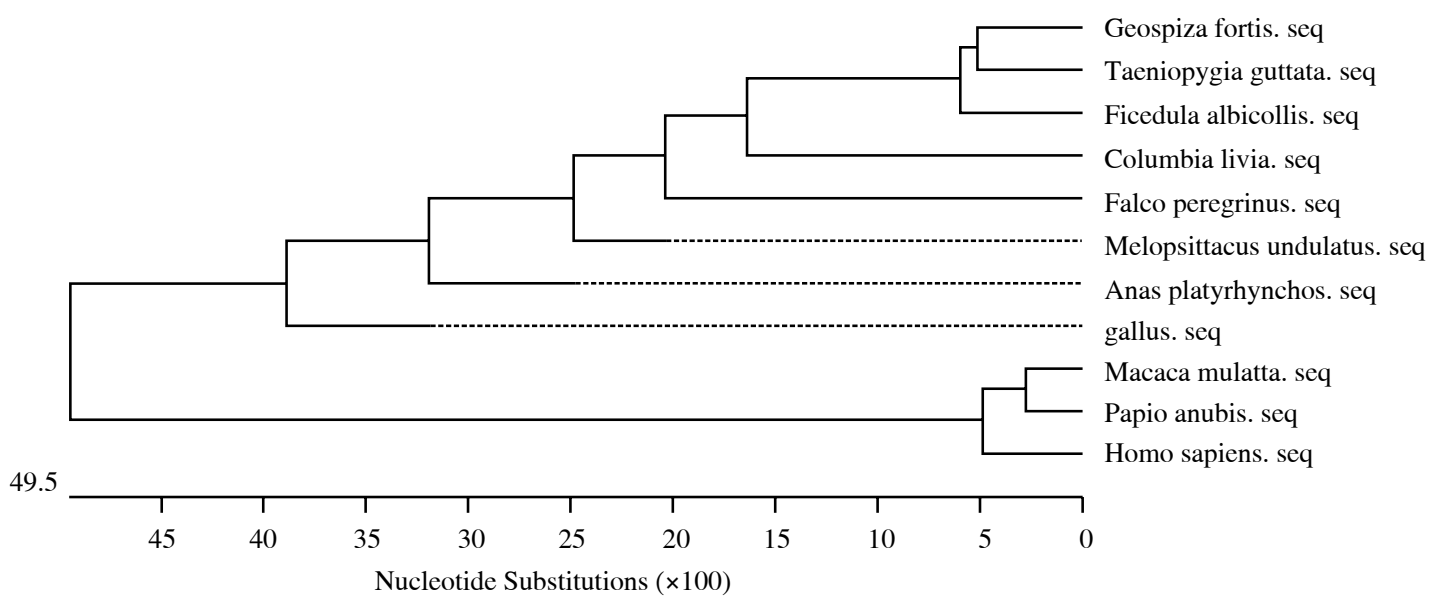

Fig. 4. The evolutionary tree of Columba livia compared with other animals from GenBank 
columba_livia.pro.seg falco_peregrinus.seq Consensus

columba livia.pro.seq talco_peregrinus.seq Consensus

columba livia.pro.seq falco_peregrinus.seq Consensus

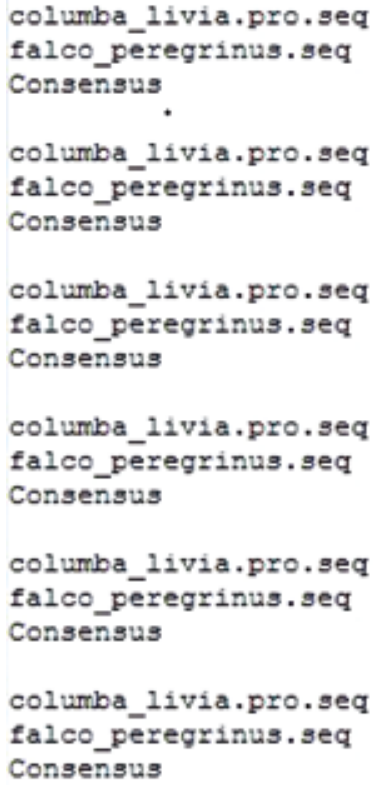

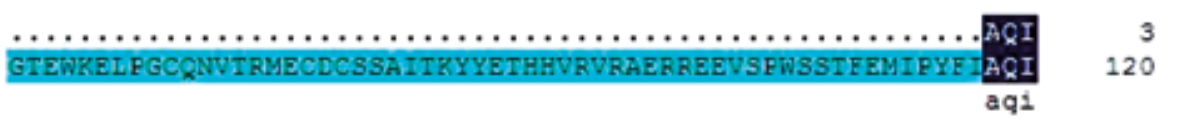

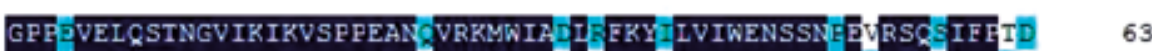

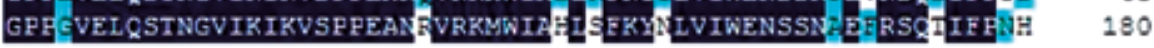
gpp velqstngvikikvgppean vrkmwia 1 fky lviwensgn e rsq ifp

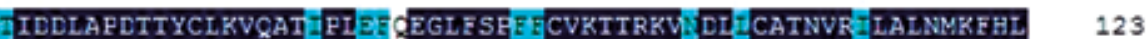 VIDDLAPDTTYCLKVQATIPIESTEGLFSEVHCVRTTRKV IDIFCATNVRVLALNMRFHL 240 iddlapdttyclkvqat pl eglfsp cvkttrkv dl catnvr lalntkfhl}

hwn ykqhv ynvqyl gy $k 1$ ddys wl vpgeen $t$ eqenfssil te $g$ yy

LRVQAMSEYNRSCLSNEVRVDPIITNEIGEFGV DVDISDVILHIQISPFGCEECEVMEDR

lrvqarse $\mathrm{nk}$ clsnevkvdpl tneigpp $\mathrm{v}$ vdisdv lniqisppgg ecevm $\mathrm{d}$

Fig. 5. Comparison of the predicted amino acid sequences of Columba livia and Falco peregrinus IFNAR-1. The blue shade reveals the conserved amino acid residues and the green shade reveals the non-conserved amino acid residues

\begin{tabular}{|c|c|c|c|c|c|c|c|c|c|c|c|c|}
\hline & $\mathbf{1}$ & $\mathbf{2}$ & $\mathbf{3}$ & $\mathbf{4}$ & $\mathbf{5}$ & $\mathbf{6}$ & $\mathbf{7}$ & $\mathbf{8}$ & $\mathbf{9}$ & $\mathbf{1 0}$ & $\mathbf{1 1}$ & \\
\hline $\mathbf{1}$ & & 69.7 & 72.7 & 57.6 & 69.3 & 61.6 & 32.5 & 34.7 & 71.2 & 39.8 & 61.7 & $\mathbf{1}$ \\
\hline $\mathbf{2}$ & 38.7 & & 80.4 & 67.1 & 68.0 & 68.5 & 36.8 & 36.4 & 77.3 & 37.1 & 68.8 & $\mathbf{2}$ \\
\hline $\mathbf{3}$ & 33.9 & 22.8 & & 66.7 & 68.5 & 66.5 & 38.8 & 39.2 & 77.8 & 40.4 & 65.7 & $\mathbf{3}$ \\
\hline $\mathbf{4}$ & 61.5 & 43.1 & 43.9 & & 59.5 & 85.7 & 30.4 & 31.8 & 64.7 & 35.0 & 80.4 & $\mathbf{4}$ \\
\hline $\mathbf{5}$ & 39.5 & 41.7 & 40.8 & 57.7 & & 60.0 & 35.7 & 36.4 & 67.0 & 37.3 & 58.8 & $\mathbf{5}$ \\
\hline $\mathbf{6}$ & 53.3 & 40.8 & 44.3 & 15.9 & 56.6 & & 32.9 & 34.3 & 65.1 & 34.2 & 83.9 & $\mathbf{6}$ \\
\hline $\mathbf{7}$ & 145.5 & 124.6 & 115.9 & 157.5 & 129.4 & 143.2 & & 83.4 & 36.7 & 91.4 & 31.2 & $\mathbf{7}$ \\
\hline $\mathbf{8}$ & 134.1 & 126.0 & 114.6 & 149.0 & 126.4 & 135.8 & 18.9 & & 36.9 & 97.3 & 32.5 & $\mathbf{8}$ \\
\hline $\mathbf{9}$ & 36.2 & 27.0 & 26.3 & 47.5 & 43.3 & 46.7 & 124.8 & 124.2 & & 38.4 & 62.0 & $\mathbf{9}$ \\
\hline $\mathbf{1 0}$ & 112.3 & 123.2 & 110.1 & 132.7 & 122.1 & 136.3 & 9.1 & 2.7 & 117.8 & & 34.6 & $\mathbf{1 0}$ \\
\hline $\mathbf{1 1}$ & 53.1 & 40.3 & 45.7 & 22.8 & 59.0 & 18.2 & 152.8 & 145.5 & 52.6 & 134.3 & & $\mathbf{1 1}$ \\
\hline & $\mathbf{1}$ & $\mathbf{2}$ & $\mathbf{3}$ & $\mathbf{4}$ & $\mathbf{5}$ & $\mathbf{6}$ & $\mathbf{7}$ & $\mathbf{8}$ & $\mathbf{9}$ & $\mathbf{1 0}$ & $\mathbf{1 1}$ & \\
\hline
\end{tabular}

Fig. 6. Homology comparisons of amino acid sequences among Gallus, Columba livia, Falco peregrinus and other animals IFNAR-1 from GenBank 


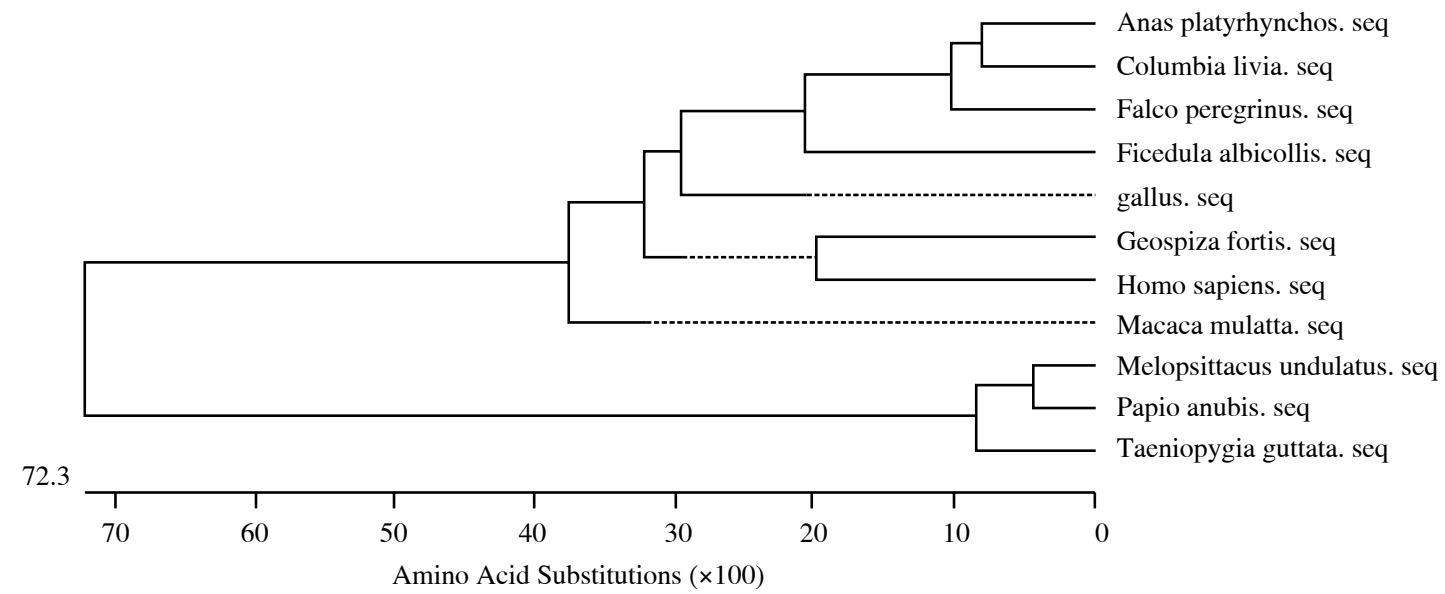

Fig. 7. The evolutionary tree of Columba livia compared with other animals from GenBank

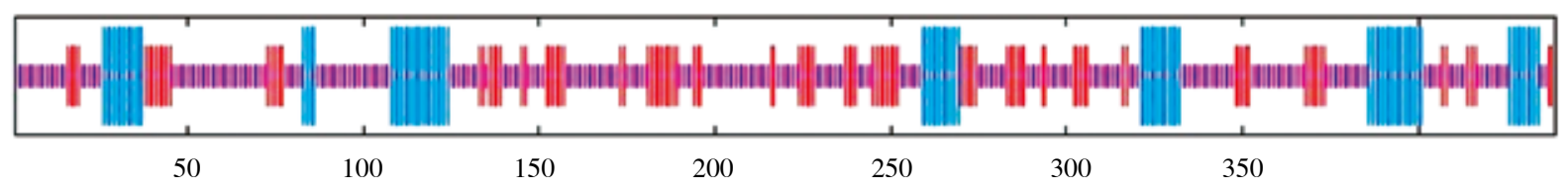

Fig. 8. The predicted secondary structure of Columba livia IFNAR-1amino acid sequence. The blue line represents $\alpha$-helix, the red line represents extended strand and the purple line represents random coil

identified from the spleen cDNA library of Columba livia, which are homologous to Gallus and Taeniopygia guttata. Based on this sequence, the 3'terminal cDNA was cloned using 3'RACE PCR. After splicing, a $1253 \mathrm{bp}$ fragment (Fig. 2) of Columba livia IFNAR-1 was obtained.

\section{IFNAR-1 sequence analysis}

The Columba livia IFNAR-1 displayed $88.5 \%, 80.5 \%$ and $73.8 \%$ nucleotide identity to Falco peregrinus, Gallus and Taeniopygia guttata, respectively (Figs. 3 and 4). Analysis of the phylogenetic tree showed that the relationship of Columba livia and Falco peregrinus IFNAR-1 had high homology.

\section{IFNAR1 amino acid analysis}

The deduced amino acid (aa) sequences of Columba livia IFNAR-1 had an estimated isoelectric point and $\mathrm{Mr}$ of 5.55 and $50.0 \mathrm{KD}$, respectively. Multiple sequence alignment was carried out using the software package MegAlign (Lasergene 7.0, DNAStar Inc., Madison, WI). The Columba livia IFNAR1 displayed $80.4 \%$ and $68 \%$ aa identity to Falco peregrinus and Gallus, respectively. The inferred aa sequences of the IFNAR-1 of Columba livia and Falco peregrinus were aligned (Fig. 5). There were few amino acid mutations between Columba livia and Falco peregrinus; therefore, they had high homology. Figures
6 and 7 show the distance-based neighbour-joining tree displaying the phylogenetic relationships among Columba livia and other animals based on the IFNARI gene. Columba livia has a closer relationship with birds such as Falco peregrinus and Gallus, and it has a more distant relationship with mammals. The secondary structure of the sequence was predicted to be mainly random coil, separated by extended strand and $\alpha$-helix (Fig. 8). Transmembrane regions were predicted to be outside between the first and

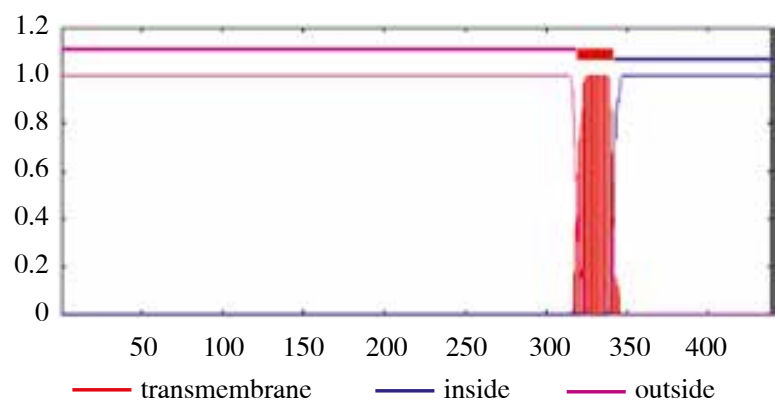

Fig. 9. Analysis of transmembrane regions of Columba livia IFNAR-1. The blue line represents inside, the red line represents transmembrane and the purple line represents outside 

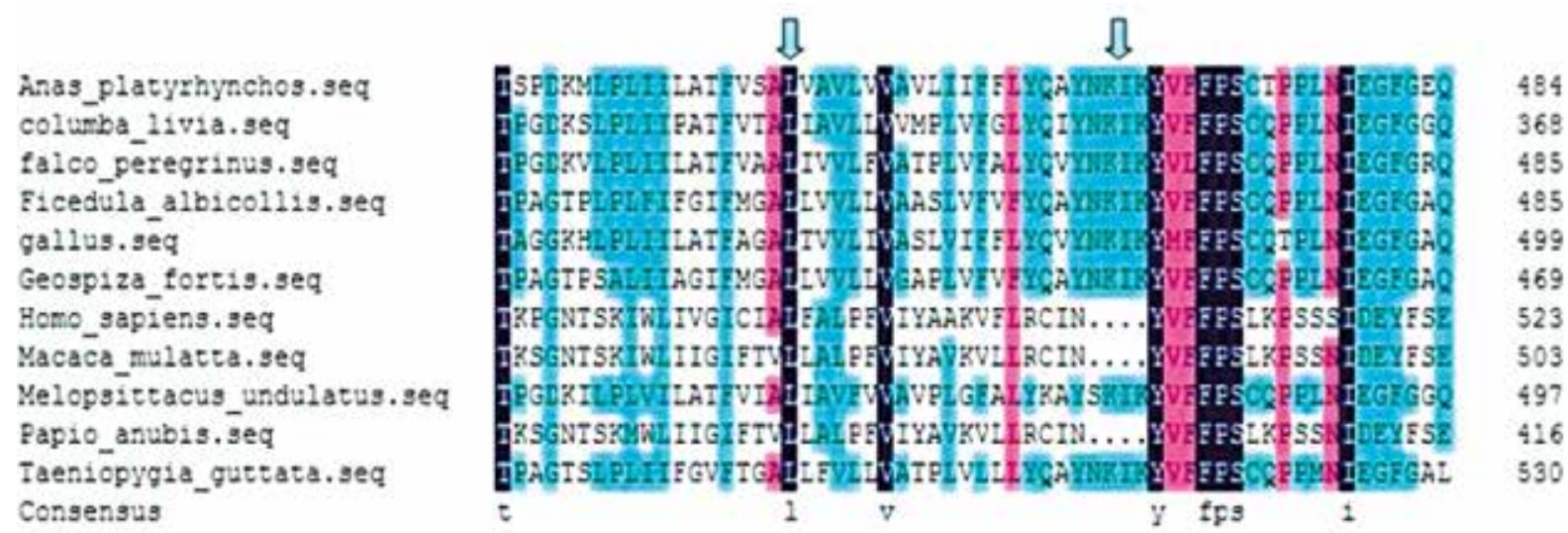

Fig. 10. Comparison of the amino acid sequences of several avian and mammalian species. The region between the two arrows represents the transmembrane region

$318^{\text {th }}$ amino acid, and transmembrane between $319^{\text {th }}$ and $341^{\text {th }}$, and inside between the $342^{\text {th }}$ and $439^{\text {th }}$ (Fig. 9).

\section{Discussion}

According to the results, we can determine that Columba livia IFNAR-1 had high homology with avian, while its identity to mammalian was low. The highest homology was with Falco peregrinus (88.5\%), the second was with Melopsittacus undulates (86\%) and the third was with Gallus $(80.5 \%)$.

According to the analysis of the amino acid sequences of the species by TMHMM Server v2.0, the transmembrane regions are similar. They all include approximately 20 amino acids that are all hydrophobic amino acids, such as Ala, Leu (Fig. 10).

The meat of Columba livia, known as "Dragon-meat", is delicious in taste and well received in China, especially as squab. The number of Columba livia being raised is about 50 million and it is worldwide in distribution. Columba livia is an important part of China's special economic animal production. But the occurrence of avian influenza came to be a serious threat to the production of Columba livia. It is necessary for Columba livia breeding to establish an immune mechanism, to find a new and an efficient security immune route. By contrast, the research of interferon receptor lags behind other areas of interferon research. In this study, we successfully obtained a Columba livia IFNAR-1 partial sequence and analysed the gene sequence and the amino acid sequences. The result can be used as reference for further research and practical application.

The authors declare no conflict of interest.

\section{References}

1. Isaacs A, Lindenmann J (1957): Virus interference. I. The interferon. Proc R Soc Lond B Biol Sci 147: 258-267.

2. Altmann SM, Mellon MT, Distel DL, Kim CH (2003): Molecular and functional analysis of an interferon gene from the zebrafish, Danio rerio. J Virol 77: 1992-2002.

3. Sen GC (2001): Viruses and interferons. Annu Rev Microbiol 55: $255-281$.

4. Shtrichman R, Samuel CE (2001): The role of gamma interferon in antimicrobial immunity. Curr Opin Microbiol 4: 251-259.

5. Colamonici OR, D'Alessandro F, Diaz MO, et al. (1990): Characterization of three monoclonal antibodies that recognize the interferon $\alpha$ receptor. Proc Natl Acad Sci U S A 87: 7230-7234.

6. Colamonici OR, Domanski P (1993): Identification of a new sub unit of the type I IFN receptor localized to human chromosome 21. J Biol Chem 268: 10895-10899.

7. Samuel CE (2007): Interferons, interferon receptors, signal transducer and transcriptional activators, and interferon regulatory factors. J Biol Chem 282: 20045-20046.

8. Taniguchi T, Takaoka A (2002): The interferon- $\alpha / \beta$ system in antiviral responses: a multimodal machinery of gene regulation by the IRF family of transcription factors. Curr Opin Immunol 14: 111-116.

9. Müller M, Briscoe J, Laxton C, et al. (1993): The protein tyrosine kinase AK1 complements defects in the interferon- $\alpha / \beta$ and $-\gamma$ signal transduction. Nature 366: 129-135. 